\title{
Human-Oriented Design of an Indoor Thermal Environment
}

\author{
Masanari Ukai $^{1}$, and Tatsuo Nobe $^{2}$ \\ ${ }^{1}$ Waseda University, Department of Architecture, Tokyo, Japan \\ ${ }^{2}$ Kogakuin University, Department of Architecture, Tokyo, Japan
}

\begin{abstract}
In this study, an initial survey of clothing insulation and changes in the metabolic rate of individuals in office spaces was performed to establish the distribution of room temperatures at which individuals perceived a neutral thermal sensation. Subsequently, the indoor thermal environment in four offices was surveyed during the summer with different air-conditioning systems to determine the thermal environment stability in each case. The results revealed that for the required temperature, there was a noticeable difference between the average and most frequent values. Moreover, it was determined that the required temperature distribution is not normal, but rather, it is skewed to the low-temperature side. In addition, the radiant air-conditioning system was found to generate a narrow distribution of the equivalent temperature and hence, facilitated a more uniform thermal environment compared to a convective (multi-unit) air-conditioning system. Therefore, in buildings with convective airconditioning systems, even if the planar average thermal environment is categorized as comfortable, it may be possible that workers who are sensitive to the cold or heat will complain of discomfort more frequently than those in buildings with radiant air-conditioning systems because the probability of workers sitting in cold- or hot-spot areas is higher in the former case.
\end{abstract}

\section{Introduction}

Conventional air-conditioning systems aim to provide indoor thermal environments of a temperature that is the average value of the group's required temperature, the required temperature being defined as the temperature at which a person in the room perceives a neutral thermal sensation. Therefore, conventional air-conditioning systems endeavour to provide comfortable and thermally uniform environments by preventing temporal and spatial variations. However, in a previous survey of thermal environment acceptability in offices, the authors recorded votes indicating an unacceptable environment even when the thermal environment was consistently maintained at comfortable levels according to the American Society of Heating, Refrigerating and AirConditioning Engineer's (ASHRAE) standards. Moreover, votes indicating an unacceptably hot environment concentrated soon after workers sat down. Conversely, votes indicating an unacceptably cold environment occurred in greater numbers when workers had been sitting for a long time [1]. The perceived comfort level of occupants is affected not only by the overall thermal uniformity of the indoor environment, but also by the difference in preferred temperature due to the variation in clothing choice and metabolic rate. In a previous study by our group, a new thermal comfort index called a P-R chart was proposed based on the concepts of a required temperature distribution such that an individual perceives a neutral thermal sensation and uniformity of the indoor thermal environment [2].
In this study, an initial survey of clothing insulation and changes in the metabolic rate of individuals in office spaces was performed to establish the distribution of room temperature at which an individual perceived a neutral thermal sensation. Subsequently, the indoor thermal environment in four offices was surveyed during the summer with different air-conditioning systems to determine the thermal environment stabilities in each case. Finally, the differences in thermal environment acceptability between the radiant and conventional convective air-conditioning systems were evaluated using a P-R chart.

\section{Concept of evaluating thermal environment acceptability using $P-R$ chart}

The authors developed a new thermal comfort index called the $\mathrm{P}-\mathrm{R}$ chart using the concepts of "provided temperature" and "required temperature" for use in evaluating uniform, high-quality indoor thermal environments and non-uniform, unsteady thermal environments.

\subsection{Provided temperature}

Provided temperature is a quantitative index of the indoor thermal environment, defined as the temperature of a hypothetical uniform thermal environment equivalent to the real environment. The conventional index of thermal comfort is a function of air temperature, mean radiant temperature, relative humidity, air speed, metabolic rate, and clothing insulation. In contrast, provided temperature is dependent on four environmental parameters and is intended to reflect the pure indoor physical thermal environment. The authors

\footnotetext{
* Corresponding author: m.ukai@kurenai.waseda.ac.jp
} 
therefore consider provided temperature to be similar to equivalent temperature. Hence, it is considered that the provided temperature distribution of an evaluated office depends on the type of air-conditioning system present. In this study, the provided temperature was assumed to be the equivalent temperature based on the Madsen method.

\subsection{Required temperature}

Required temperature is defined as the provided temperature at which a person in the room perceives a neutral thermal sensation. Therefore, required temperature is dependent on the metabolic rate and clothing insulation of the person. Hence, the required temperature distribution of an evaluated office is considered to depend on factors such as the type of business activity occurring, clothing regulations, and gender ratio. In this study, the required temperature was assumed to be the operative temperature at which $\mathrm{PMV}=0$, based on the metabolic rate and clothing insulation.

\subsection{P-R chart}

The proposed index employs the provided temperature and required temperature and is hence termed a $\mathrm{P}-\mathrm{R}$ chart. The values used are all assumed, and conceptual diagrams are shown in Figure 1. This approach applies probabilistic evaluation to thermal acceptability. The thermally neutral line (shown in white on the charts) indicates the points at which the provided temperature and required temperature are equivalent. Therefore, a person whose thermal preference is represented by the white line feels that the thermal environment is acceptable (neither too hot nor too cold). In contrast, the authors considered a case in which $20 \%$ of workers preferred a low temperature because their metabolic rate and clothing insulation were high. Assuming that the provided temperature in the indoor thermal environment is $10 \%$ skewed toward the hot side, in evaluating the indoor thermal environment using this concept, it can be probabilistically determined that $2 \%$ of workers feel that the thermal environment is too hot. Moreover, in the case of an indoor thermal environment with a radiant air-conditioning system, the probability of worker complaints decreases because the maldistribution of provided temperature becomes lower.

In this study, the authors first performed an experiment to clarify the distribution of the required temperature in typical offices. Subsequently, the authors measured the indoor thermal environment stabilities in four offices with different air-conditioning systems in order to clarify the distribution of the provided temperature in typical offices. Finally, the differences in the thermal environment acceptability between the radiant air-conditioning systems and conventional convective air-conditioning systems were evaluated using the $\mathrm{P}-\mathrm{R}$ chart.
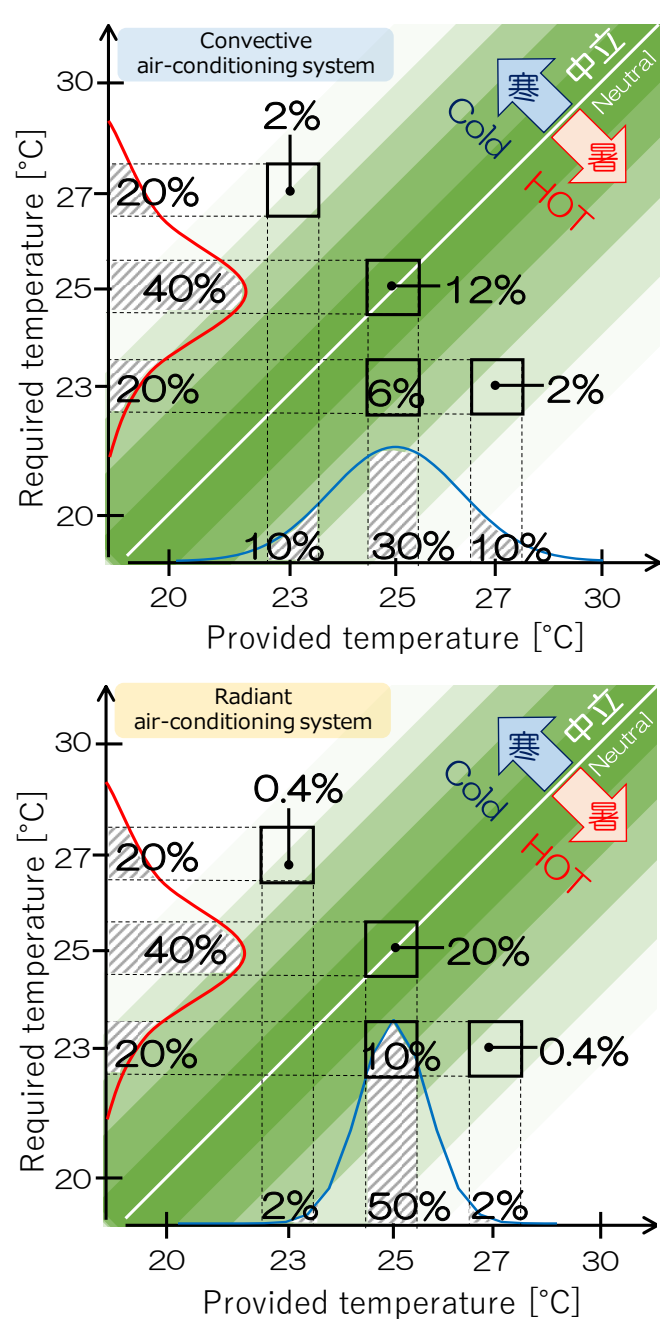

Fig. 1. Concept diagram of the $\mathrm{P}-\mathrm{R}$ chart.

Table 1. Physical activity meter.
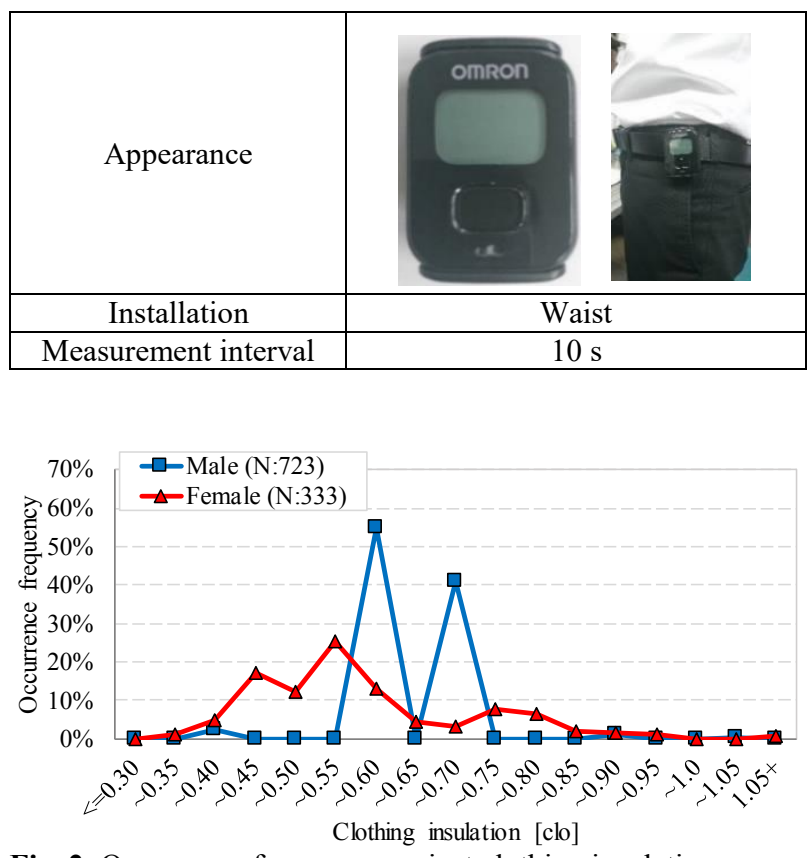

Fig. 2. Occurrence frequency against clothing insulation. 


\section{Investigation of required temperature distribution}

\subsection{Measuring method}

The authors surveyed clothing insulation and changes in metabolic rate for individuals in offices and sought to calculate a required temperature distribution for workers in summer. The clothing rate was evaluated via a questionnaire survey of 1590 workers in nine offices. The metabolic rates of 86 workers in three offices were measured using a physical activity meter (Table 1).

\subsection{Clothing insulation of office workers}

Figure 2 illustrates the occurrence frequency of clothing insulation in summer for males and females. Clothing insulation refers to the typical guidelines calculated from a thermal manikin in AIJES-H0005-2015 [2]. However, the clothing insulation of the assemblage not stated in AIJESH0005-2015 was calculated via Equations 1 or 2 using the sum of the clothing insulation of each body part [4].

In the case of male clothes,

$$
I_{\text {clo }}=0.708 \Sigma I_{\text {clo }, i}+0.052
$$

In the case of female clothes,

$$
I_{\text {clo }}=0.828 \Sigma I_{\text {clo }, i}+0.013
$$

where $I_{c l o}$ is the clothing insulation of the entire body and $I_{c l o, i}$ is the clothing insulation of the $i$-th part of the body.

The clothing distribution for male workers has two peaks corresponding to a short-sleeve shirt style $(0.56$ clo $)$ and a long-sleeve shirt style $(0.68 \mathrm{clo})$. The clothing distribution of female workers exhibits more peaks because female workers have a wider range of clothing options. Moreover, females tended to prefer clothing with lower insulation values than males.

\subsection{Metabolic rate of office workers}

The physical activity meter used in this survey determined the measured exercise intensity $\left(\mathrm{MET}_{\mathrm{s}}\right)$, a metric often used in sports medicine. Both $\mathrm{MET}_{\mathrm{s}}$ and the metabolic rate (met) metric used by architects are defined in terms of ratios to the resting metabolic rate (RMR) met is defined based on the portion of the RMR that contains diet-induced thermogenesis (DIT). However, MET ${ }_{\mathrm{s}}$ is defined as the part of the RMR that does not include DIT. The RMR that contains DIT is 1.2 times the basal metabolic rate (BMR), whereas the RMR that does not contain DIT is 1.1 times the BMR. Moreover, the typical RMR per body surface area $\left(\mathrm{RMR}_{\mathrm{s}}\right)$ is $58.2 \mathrm{~W} / \mathrm{m}^{2}$. Alternatively in this study, the authors used the Japanese average $R M R_{s}$ value of $50.0 \mathrm{~W} / \mathrm{m}^{2}$. Therefore, the relationship between $\mathrm{MET}_{\mathrm{s}}$ and met is realized by the following equation:

$m e t=M E T_{s} \times \frac{1.1}{1.2} \times \frac{50.0}{58.2}$

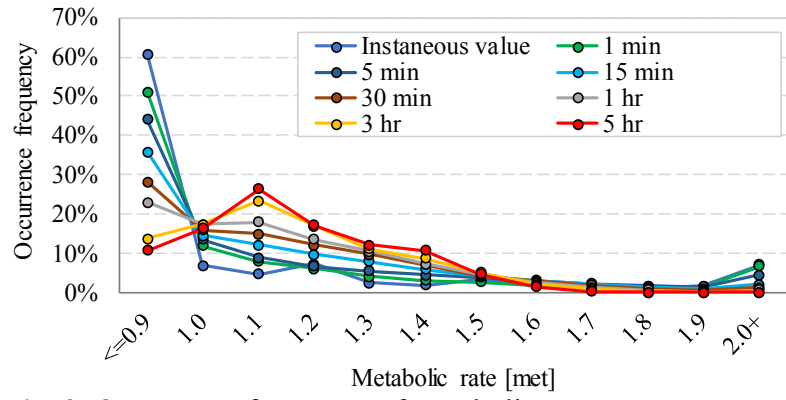

Fig. 3. Occurrence frequency of metabolic rate.

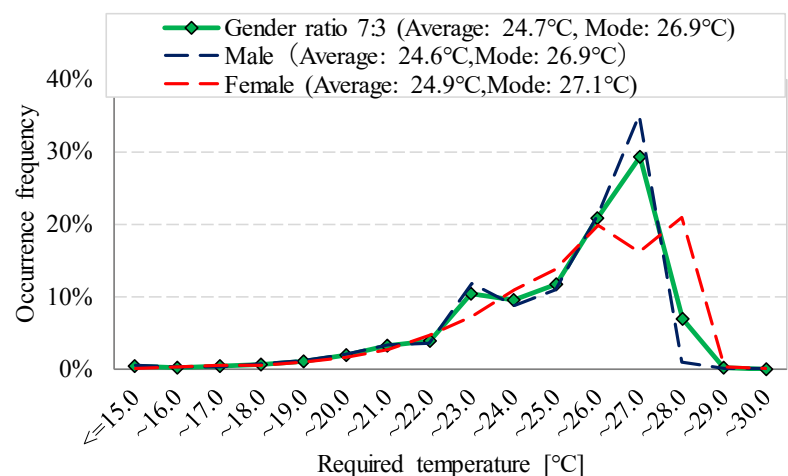

Fig. 4. Occurrence frequency of required temperature.

Figure 3 shows the occurrence frequency of workers' metabolic rate, from the instantaneous value to a 5-hour movement average during business hours. The movement average term has a longer duration, and the distribution of metabolic rate is most concentrated at 1.1 met. This result corresponds to conventional data stating that the metabolic rate of office work is 1.1 met. However, workers whose metabolic rates were low and those whose metabolic rates were high were mixed in the same offices.

\subsection{Required temperature distribution}

The authors defined the required temperature as the temperature at which a person in the room perceived a neutral thermal sensation. In this study, the required temperature was assumed to be the operative temperature at which $\mathrm{PMV}=0$. Therefore, the required temperature distribution was calculated from the measured clothing insulation (shown in Figure 2) and metabolic rate (shown in Figure 3, assuming a movement average value of $15 \mathrm{~min}$ ), and assuming a $0.1 \mathrm{~m} / \mathrm{s}$ air speed and 50\% relative humidity. Figure 4 shows the distribution of the required temperature. The required temperature for females tended to be higher than that of males because females tended to prefer clothing with lower insulation values than males. The green line shows the required temperature distribution in a standard office room for an assumed male-to-female ratio of 7 to 3 . The shape of the required temperature distribution is asymmetric, being very broad on the lower side. Moreover, there is a noticeable difference between the average value $\left(24.7^{\circ} \mathrm{C}\right)$ and the modal value $\left(26.9^{\circ} \mathrm{C}\right)$. 


\section{Investigation of provided temperature distribution in offices with different air- conditioning systems}

\subsection{Measurement method}

This survey was conducted to characterise indoor thermal environment uniformity variations for various airconditioning systems using the provided temperature distribution. In this study, the evaluation of the provided temperature distribution was performed using Madsen's technique for evaluating the equivalent temperature (Equation 4) on a trial basis [5].

$$
\begin{aligned}
t_{e q}=0.55 \times t_{a}+ & 0.45 \times t_{r} \\
& +\frac{0.24-0.75 \sqrt{v_{a}}}{1+I_{c l}}\left(36.5-t_{a}\right)
\end{aligned}
$$

where $t_{e q}$ is the equivalent temperature $\left[{ }^{\circ} \mathrm{C}\right], t_{r}$ is the mean radiant temperature $\left[{ }^{\circ} \mathrm{C}\right]$, and $I_{c l}$ is the clothing insulation [clo] (assumed to be 0.5 [clo]).

The planar indoor thermal environment was measured during the summer in four offices with different air-conditioning systems. The conditions for each measurement location are shown in Figure 5. Office $\mathrm{N}$ employs a radiant airconditioning system with a combination of systems known as a thermally activated building system (TABS). TABS is a building component in which embedded pipes containing water are used to cool and heat the building. Office T employs a floor-supply displacement heating, ventilation, and air conditioning (HVAC) system to control the temperature and humidity inside the office. In this system, cold drafts and nonuniform thermal environments do not occur due to the air speed being very slowly and air passing through the carpeted floor. Offices A and K employ a convective air-conditioning system known as a ceiling-concealed-type multiunit airconditioning system. In offices A and $\mathrm{K}$, remote controllers are used to operate the air conditioners. In office $\mathrm{K}$, workers can change the set temperature and the air volume freely. In office A, workers can change only the set air volume. The device used to measure the thermal environment (Table 2) can record the air temperature, globe temperature, and air speed. The locations of the sensors are shown in Figure 5.

\subsection{Provided temperature distribution}

Figure 6 shows the occurrence frequency of the provided temperature during operational hours. Figure 7 shows the occurrence frequency of divergence between the target set value of the provided temperature during operational hours and the instantaneous value of the local provided temperature. The data shown in Figure 7 is divided into an air-conditioning system group that is capable of providing a thermal environment with high uniformity and a conventional convective air-conditioning group. The high uniformity thermal environment group is the average of office $\mathrm{N}$ and office $\mathrm{T}$. The convective air-conditioning group is the average of office $\mathrm{K}$ and office $\mathrm{A}$.
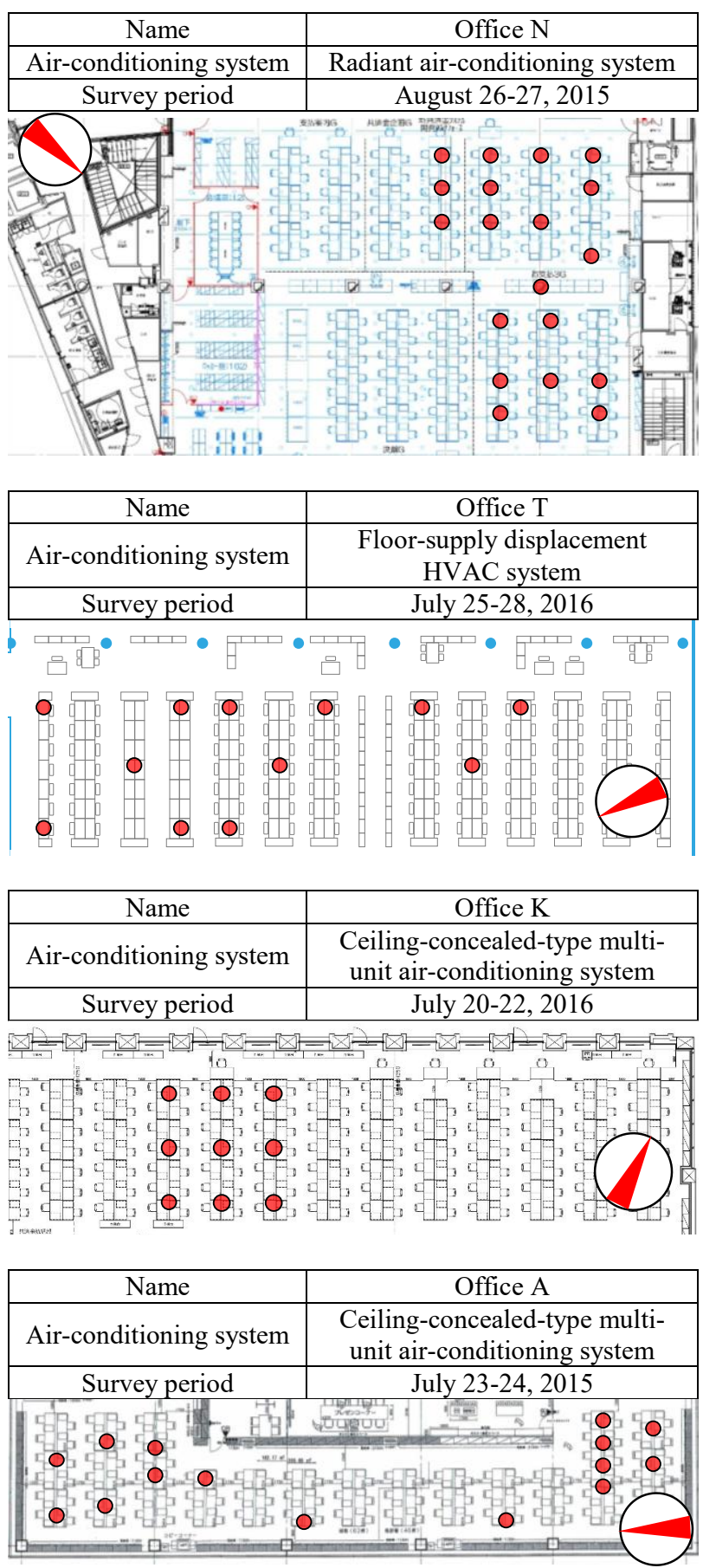

Fig. 5. Floor plan and thermal measurement points for each office.

Table 2. Details of device used to measure the thermal environment.

\begin{tabular}{|c|c|}
\hline Appearance & Personal desk \\
\hline Installation & $\begin{array}{c}\text { Air temperature: } 5 \text { min, Globe } \\
\text { temperature: } 5 \text { min }\end{array}$ \\
\hline $\begin{array}{c}\text { Measurement } \\
\text { interval }\end{array}$ & $\begin{array}{c}\text { Relative humidity: } 5 \text { min, Air speed: } \\
1 \text { min }\end{array}$ \\
\hline
\end{tabular}


The radiant air-conditioning system and the floor-supply displacement HVAC system achieve a narrow distribution of the provided temperature and hence provide more uniform thermal environments than the convective (multiunit) airconditioning system.

\section{Evaluation of thermal environment acceptability using $P-R$ chart}

Figure 8 shows the $\mathrm{P}-\mathrm{R}$ chart results for an indoor thermal environment with a convective air-conditioning system (upper panel) and a highly uniform indoor thermal environment such as one using a radiant air-conditioning system (lower panel). The evaluation of the provided temperature distribution was performed using Figure 7 on a trial basis. On the other hand, the evaluation of the required temperature distribution was conducted in a standard office room by assuming that the ratio of males to females was 7 to 3 , as shown in Figure 4, on a trial basis. A highly uniform indoor thermal environment such as one using a radiant airconditioning system had many workers near the thermally neutral line (i.e., the provided temperature being near the required temperature of the workers) because the provided temperature range was narrow. On the other hand, for the convective air-conditioning system, the provided temperature for many workers was different from the required temperature because the provided temperature range was broad. Figure 9 shows the occurrence frequencies for the divergence between provided temperature and required temperature from the $\mathrm{P}-\mathrm{R}$ chart. The shape of the distribution of the divergence between the provided and required temperatures is asymmetric and very broad on the lower side. Therefore, even if many workers feel comfortable with the indoor thermal environment, it may be possible that complaints of being too hot will occur more frequently than those of being too cold. However, for the convective airconditioning system, the divergence between the provided and required temperatures is distributed toward the hot side compared with that of the highly uniform thermal environment. Therefore, it may be possible that workers who are sensitive to colder temperatures will complain of discomfort more frequently than those in buildings with a radiant air-conditioning system, because the probability of workers sitting in cold-spot areas generated by the air outlets is higher in the former case.

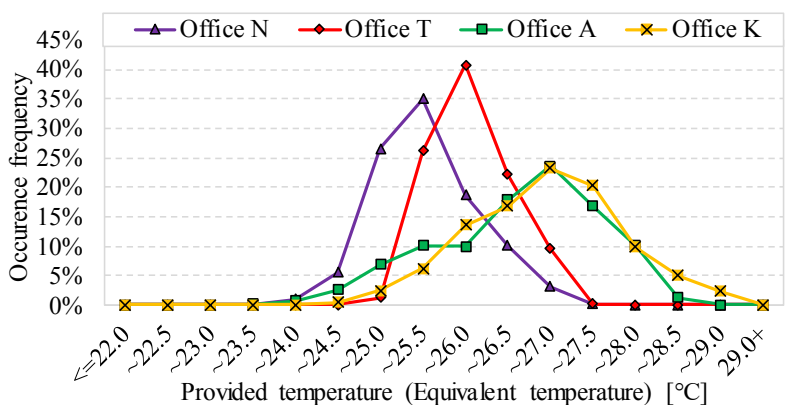

Fig. 6. Occurrence frequency of provided temperature (equivalent temperature).

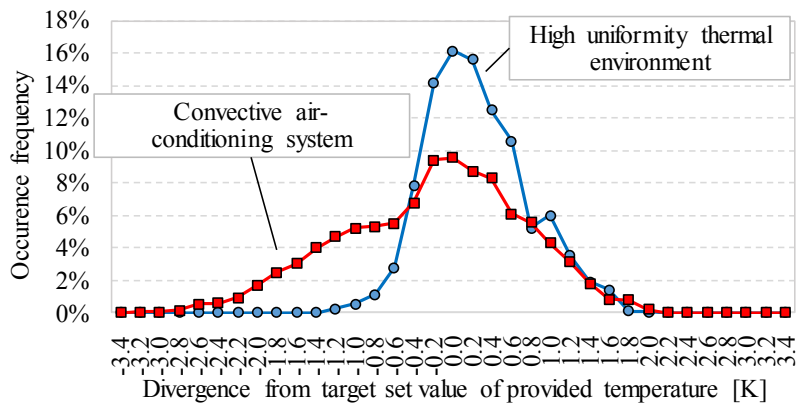

Fig. 7. Divergence from target set value of provided temperature.

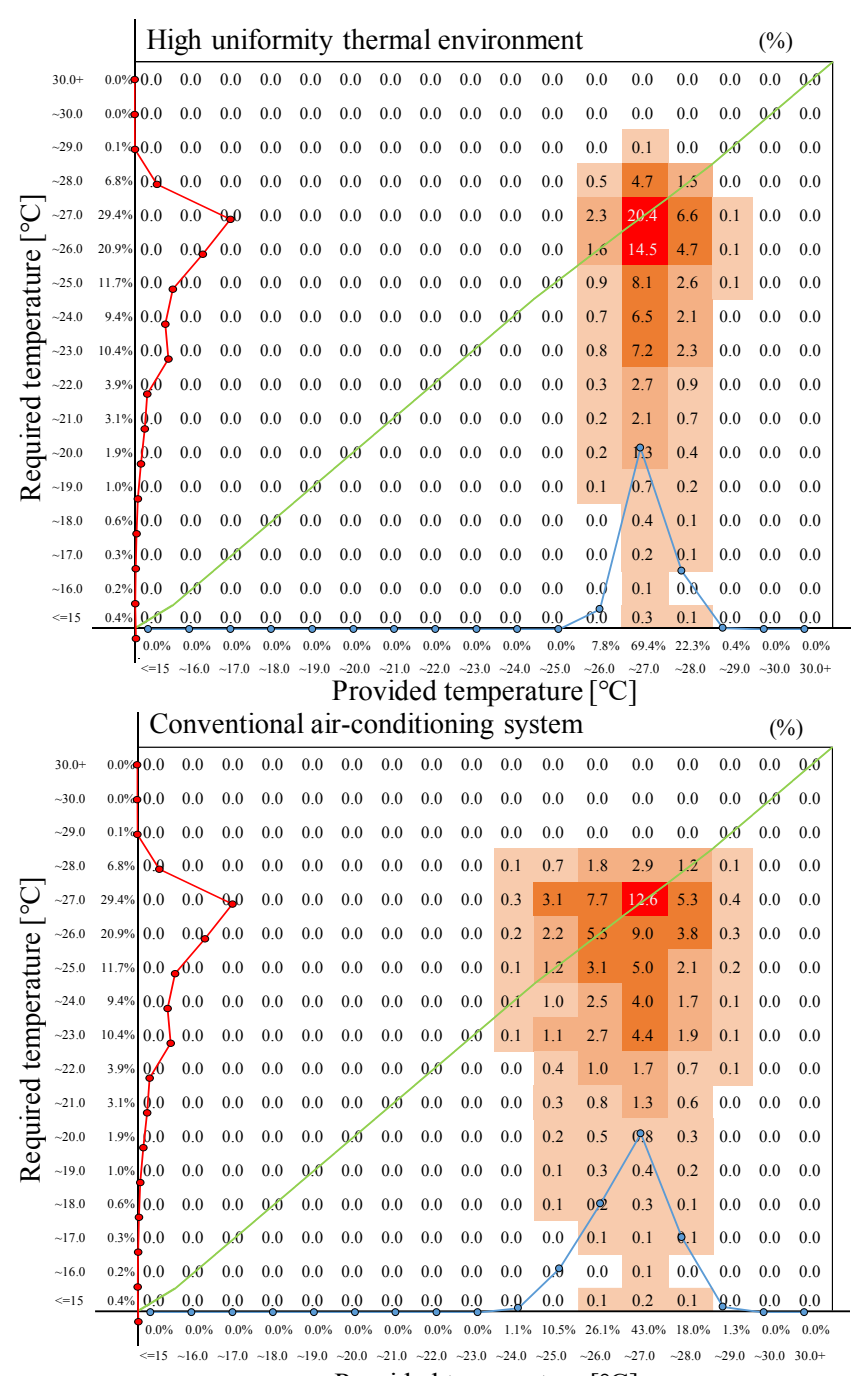

Provided temperature $\left[{ }^{\circ} \mathrm{C}\right]$

Fig. 8. Result of $\mathrm{P}-\mathrm{R}$ chart.

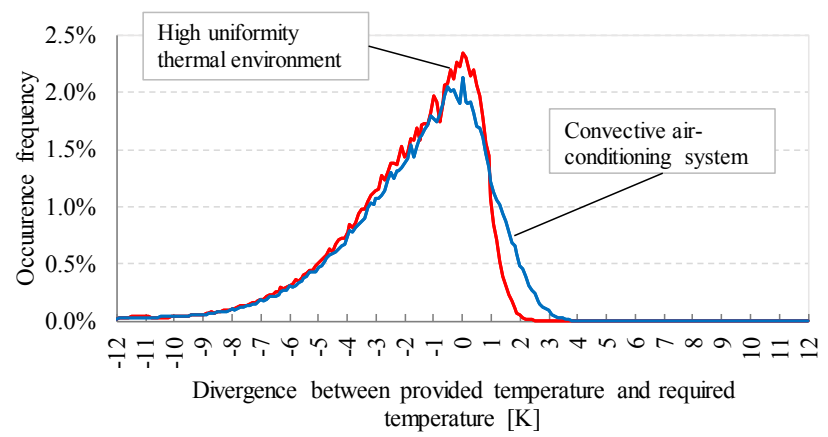

Fig. 9. Divergence between provided temperature and required temperature. 


\section{Conclusions}

In this study, the differences in the thermal environment acceptability between radiant air-conditioning systems and conventional convective air-conditioning systems were evaluated using $\mathrm{P}-\mathrm{R}$ charts. To achieve these results, the authors first surveyed the required temperature distribution for the workers in the offices. Then, the indoor thermal environment in four offices was surveyed during the summer with different air-conditioning systems to determine the provided temperature distribution in each case. These results can be summarized as follows:

1) The shape of the required temperature distribution is asymmetric and very broad on the lower side. Moreover, there is a noticeable difference between the average value $\left(24.7^{\circ} \mathrm{C}\right)$ and the modal value $\left(26.9^{\circ} \mathrm{C}\right)$.

2) The radiant air-conditioning system and the floorsupply displacement HVAC system achieve a narrow distribution of the provided temperature and hence provide more uniform thermal environments than the convective (multiunit) air-conditioning system.

3) The shape of the distribution of the divergence between the provided and required temperatures is asymmetric and very broad on the lower side. Therefore, even if many workers feel comfortable with the indoor thermal environment, it may be possible that complaints of being too hot will occur more frequently than those of being too cold.

4) For the convective air-conditioning system, divergence between the provided and required temperatures was distributed further towards the hot side compared to that of highly uniform thermal environments such as those generated by using radiant air-conditioning systems.

\section{References}

1. Ukai, M. et al, 2014. Field Study of Thermal Environment Acceptability in Offices Using the Ostracon Voting Device and Seat Occupancy Sensors. Grand Renewable Energy 2014 Proceedings. Tokyo, Japan, 27 July-1 August 2014 (in English)

2. Ukai, M. et al, 2016. A Study on Probabilistic Thermal Acceptability Evaluation. Proceedings of 9th Windsor Conference. Windsor, UK, 7-10 April 2016 (in English).

3. AIJ. (2015). AIJES H0005-2015-Standards for Evaluation of Indoor Thermal Environment using Thermal Manikin.

4. SHASE. (2006). Mechanism of Comfortable Thermal Environment.

5. Madsen T. L. et al, 1984. Comparison Between Operative and Equivalent Temperature Under Typical Indoor Conditions. ASHRAE Transactions, 90 (I), pp 1077-1090. 